\title{
INTRACRYSTALLINE BIOMOLECULES IN RECENT AND FOSSIL BRACHIOPOD SHELLS - THE BIOCHEMISTRY OF SHELL COLORATION
}

CLEGG*, Heather, Dept. of Geology, Lilybank Gardens, Glasgow University, Glasgow, Scotland; CURRY, Gordon, B., Dept. of Geology, Glasgow University, Glasgow, Scotland.

The study of fossil biomolecules requires not only well preserved fossil samples, but abundant Recent material so that reference points can be established. Ideally it would also be possible to study the in situ decay of organic molecules in samples of different geological ages, and hence determine how degradation proceeds with time. The shells of brachiopods fulfill both these criteria, containing up to $1 \%$ organic components entombed within their shells, which are composed of highly stable, low magnesium calcite, that survives well in the fossil record for hundreds of millions of years.

This particular study has concentrated on Plio-Pleistocene brachiopods from New Zealand. These fossils are the ancestors of living species, and hence it is possible to investigate intracrystalline biomolecules in modern day shells, as well as the degradation products of such biomolecules in fossil shells of the same species.

Three of the species investigated have bright red shells in life, and during the study it became clear that this coloration was caused by a carotenoprotein which was embedded within, rather than around, the calcite fibres of the brachiopod shell. Carotenoproteins are composite molecules composed of a protein and a prosthetic carotenoid group. The protein has been characterised by hplc (high performance liquid chromatography) and SDS PAGE (sodium dodecylsulphate polyacrylamide gel electrophoresis), and the amino acids at the $\mathrm{N}$-termini have been sequenced, demonstrating that this is a small protein which has a molecular weight of $6.5 \mathrm{kDa}$ and is identical in all 3 species.

At least two different types of carotenoids were detected after initial purification, and these were characterised using direct probe mass spectra, and hplc/mass spectra. Using these techniques the two carotenoids have been tentatively identified as canthaxanthin and the mono acetylenic analogue of asthaxanthin.

In addition to the carotenoproteins, the calcite crystals of recent brachiopod shells contain lipids (carboxylic acids) and carbohydrates, which were characterised by gas chromatography (GC) and gas chromatography/mass spectrometry analyses (GC/MS).

Fossil brachiopod shells could be distinguished from recent shells due to their lack of unsaturated acids and smaller amounts of carboxylic acids present. However there was no trend of decreasing quantities of fatty acid with increasing age.

The presence of organic molecules such as carotenoproteins, lipids and carbohydrates within calcite crystals poses two questions. Firstly, are these compounds merely wastes metabolic products and are deposited within the shell during growth or, secondly, do they play an important role in the process of biomineralisation? At present these questions remain largely unanswered, because at present only the function of the carotenoprotein has been determined from the spectrum of biomolecules present within the shell (and the carotenoprotein may have other functions apart from coloration). 